\title{
Construindo-se terapeuta ocupacional no grupo de reflexão da prática: um espaço para ação-reflexão-ação
}

\section{Constructing an occupational therapist in the reflective practice group: a space for action-reflection-action}

\author{
Regina Helena Vitale Torkomian Joaquim¹, Taís Quevedo Marcolino², \\ Maria Fernanda Barboza $\mathrm{Cid}^{3}$
}

http://dx.doi.org/10.11606/issn.2238-6149.v28i2p254-260

\begin{abstract}
Joaquim RHVT, Marcolino TQ, Cid MFB. Construindo-se terapeuta ocupacional no grupo de reflexão da prática: um espaço para ação-reflexão-ação. Rev Ter Ocup Univ São Paulo. 2017 maio-ago.;28(2):254-60.

RESUMO: A formação em Terapia Ocupacional demanda diversificação de cenários e estratégias de ensino-aprendizagem. No terceiro ciclo da unidade de prática na UFSCar os estudantes subdividem-se em grupos de reflexão da prática, em processo de metodologia ativa, ancorada na espiral construtivista. O presente trabalho relata tal experiência por meio da análise temática dos registros docentes. Os temas abarcam (a) reflexões iniciais: acolhimento e questões institucionais; (b) compreensão das pessoas sob cuidado em terapia ocupacional; (c) lugar do campo/ serviço/instituição; (d) singularidades dos sujeitos; (e) reflexões sobre ser terapeuta ocupacional; (f) responsabilidades, conceitos e instrumento de trabalho. Tal espaço para reflexão distancia-se das demandas urgentes da prática, gerando satisfação entre os estudantes, que percebem seus sentimentos, conceitos e valores que devem ser revisitados e, algumas vezes, transformados para o exercício da prática profissional.
\end{abstract}

DESCRITORES: Terapia ocupacional/ensino; Educação; Ensino/recursos humanos; Prática profissional; Capacitação de recursos humanos em saúde.
Joaquim RHVT, Marcolino TQ, Cid MFB. Constructing an occupational therapist in the reflective practice group: a space for action-reflection-action. Rev Ter Ocup Univ São Paulo. 2017 May-Aug.;28(2):254-60.

\begin{abstract}
Occupational Therapy training demands diversification of scenarios and teaching-learning strategies. In the third cycle of the practice unit at UFSCar, students are subdivided into reflective practice groups, in the process of active methodology, anchored in the constructivist spiral. This study reports this experience through the thematic analysis of the teaching records. Topics include (a) initial reflections: welcoming reception and institutional issues; (b) understanding of the people undergoing care in occupational therapy; (c) place of field/service/institution; (d) the singularities of the subjects; (e) reflections on being an occupational therapist; (f) responsibilities, concepts, and tools of work. Such space for reflection distances itself from the urgent demands of the practice, which satisfies the students, who notice their feelings, concepts, and values that must be revisited and, sometimes, modified for the exercise of the professional practice.
\end{abstract}

KEYWORDS: Occupational therapy/education; Education; Teaching/human resources; Professional practice; Health human resource training.

1. Professora Associada do Departamento de Terapia Ocupacional da Universidade Federal de São Carlos - UFSCar. E-mail: regin@ufscar. brou joaquimrhvt@gmail.com

2. Professora Adjunta do Departamento de Terapia Ocupacional da Universidade Federal de São Carlos - UFSCar. E-mail: taisquevedo@ gmail.com

3. Professora Adjunta do Departamento de Terapia Ocupacional da Universidade Federal de São Carlos - UFSCar. E-mail: mariafernandacid@gmail.com

Endereço para correspondência: Profa. Dra. Regina Helena Vitale Torkomian Joaquim. Universidade Federal de São Carlos. Departamento de Terapia Ocupacional. Rodovia Washington Luís, k. 235, São Carlos, SP, Brasil. CEP: 13565-905. E-mail: regin@ufscar.br ou joaquimrhvt@ gmail.com 


\section{INTRODUÇÃO}

$\mathrm{O}$ projeto pedagógico do curso de graduação em Terapia Ocupacional da Universidade Feceral de São Carlos (UFSCar) está centrado na oferta de experiências educativas que fomentam a construção de conhecimento teórico-prático sustentado pela prática reflexiva ${ }^{1}$ em sua inserção no campo da saúde, educação, assistência social, jurídico-social, trabalho, bem como nas práticas intersetoriais ${ }^{2,3}$.

A formação inicial em Terapia Ocupacional passa pela construção de conhecimento, habilidades e atitudes que (a) incorporem, teórica e praticamente, a relação e a compreensão entre indivíduo, suas atividades cotidianas e a inserção do cuidado em terapia ocupacional no contexto, (b) adquiram domínio do processo de terapia ocupacional do ponto de vista do raciocínio clínico-profissional, bem como da capacidade de discernimento do contexto de aplicação desse conhecimento e (c) sustentem o trabalho, de modo ético, com a clientela e seus familiares, com a rede de suporte pessoal e intersetorial e com a comunidade ${ }^{4-6}$.

Além disso, demanda-se uma diversificação de cenários de ensino-aprendizagem, que favoreçam ampla participação, dos estudantes e docentes, na rede de serviços do município, cumprindo assim uma missão fundamental de contribuir para a construção de novas práticas com impacto social ${ }^{4,6}$. O atual currículo é constituído por eixos de conhecimento teórico-prático e um deles, o eixo de Prática Simulada e Supervisionada em Terapia Ocupacional - PSSTO, foi o que menos se alterou na reestruturação curricular ocorrida em 2015, que abandonou o currículo integrado centrado em metodologias ativas de aprendizagem $^{6}$ para a construção de um projeto híbrido ${ }^{3}$.

O eixo PSSTO se desenvolve gradualmente, desde as séries iniciais do curso, em atividades de complexidade crescente - observação, prática assistida e a prática autônoma supervisionada nas diferentes áreas, instituições e níveis de atuação ${ }^{7}$ - e reflexão da prática, a partir das questões emergentes, e não mais por conteúdos, tal como na formação tradicional ${ }^{8}$, buscando solidificar a identidade profissional e a habilitação técnica, ética e pessoal do estudante. No terceiro ciclo do eixo PSSTO, os estudantes inserem-se em diferentes campos de atuação (saúde mental e reabilitação física adulto e infantojuvenil, enfermaria geral adulto e pediátrica em hospital geral, e campo social) e são subdivididos em Grupos de Reflexão da Prática/ GRP. Cada grupo contém, no máximo, 10 estudantes, representantes de todos os campos, considerando os cursos de vida. Desse modo, são dois grupos para "Infância e Juventude" e dois grupos para "Adulto e Idoso". O estudante vivencia dois campos ao longo do ano, um de cada ciclo de vida, com quatro horas de atividades práticas sob a supervisão de preceptor-docente, e duas horas no GRP, com um facilitador-docente.

A metodologia utilizada no GRP é a Espiral Construtivista $^{8}$. Esta, como uma das metodologias ativas de aprendizagem, caracteriza-se pela construção do conhecimento em seis ciclos. Com enfoque na problematização, parte da experiência vivida pelos estudantes e dos saberes prévios, estimulando atitudes críticas e reflexivas sobre essa experiência de modo que, pelo reconhecimento da dúvida e da incerteza, questões de investigação possam ser formuladas e respondidas à luz de evidências científicas ${ }^{8}$.

A Espiral Construtivista, influenciada pela Aprendizagem Baseada em Problemas, trabalha sobre eventos reais, ativando conhecimentos prévios dos estudantes e estimulando a construção colaborativa de conhecimento, a partir de situações-problema, textuais ou simuladas, e na Problematização, que se mostra como uma metodologia ativa menos controlada, pois parte da vivência de situações reais e que demanda intervenções na prática, primando pelo ciclo ação-reflexão-ação. Desse modo, a Espiral Construtivista possibilita a construção de conhecimento por meio de uma diversidade de disparadores (situações-problema textuais, simuladas ou reais) favorecendo o ensino-aprendizagem sob diferentes perspectivas 8 .

No GRP, embora os estudantes estejam inseridos em cenários reais de prática profissional, os disparadores usualmente utilizados são narrativas escritas sobre a prática, mas com temáticas pertencentes ao conteúdo programático da disciplina: 1. Sujeito e população-alvo em terapia ocupacional; 2. Identificação de necessidades em terapia ocupacional; 3. Ações/procedimentos em terapia ocupacional. A depender das reflexões no grupo, que caminha de forma singular, podem haver desdobramentos temáticos.

A partir da reflexão disparada, constrói-se uma síntese provisória (hipóteses a partir do conhecimento prévio dos estudantes) e questões de aprendizagem são elaboradas de modo a possibilitar a avaliação de tais hipóteses. Os estudantes têm uma semana para responder às questões utilizando material academicamente qualificado ou entrevistas e observações direcionadas, a depender da questão. No encontro seguinte, as questões são discutidas e elabora-se uma síntese final com a contribuição de todos os participantes o que, segundo os estudantes, permite a compreensão mais ampliada e integrada do que foi estudado, tornando a aprendizagem significativa9 . 
Destacamos, ainda, que ao final de cada encontro, faz-se uma avaliação do trabalho grupal, buscando destacar os pontos favoráveis e os que precisam melhorar sobre a participação de cada um, inclusive a do(a) facilitador(a). Como ressaltaram Cardoso et al. ${ }^{9}$, o modo de trabalhar do facilitador é central para a proposta metodológica, favorecendo a comunicação e fomentando a reflexão e a construção de conhecimento de modo colaborativo.

Desse modo, o presente trabalho busca relatar a experiência que vem sendo desenvolvida com os alunos do $3^{\circ} \mathrm{e} 4^{\circ}$ ano de graduação nos GRP, destacando as principais reflexões oriundas da prática, bem como as questões de aprendizagem geradas no processo colaborativo.

\section{METODOLOGIA}

As autoras, a partir de seu trabalho como facilitadoras do GRP, realizaram uma sistematização das anotações de seus registros nos cadernos da disciplina buscando elucidar temáticas recorrentes na reflexão dos estudantes a partir de determinados disparadores e como tal reflexão se expressava em questões de aprendizagem. Tais registros, utilizados pelas docentes-facilitadoras para sustentar seu processo de ensino durante o GRP, continham informações sobre (a) a sequência da construção de conhecimento por meio da contribuição de cada estudante às sínteses provisória e final, (b) as questões de aprendizagem elaboradas, (c) a avaliação do trabalho colaborativo de cada dia.

\section{Resultados: os conteúdos das reflexões no GRP}

As seis temáticas identificadas abordaram (1) as reflexões iniciais: o campo - do acolhimento às questões institucionais; (2) as pessoas sob cuidado em terapia ocupacional: expectativas para a vida, expectativas familiares e sociais, aspectos emocionais, enfrentamento de situações adversas, impacto no cotidiano; (3) onde estou? O lugar do campo/serviço/instituição; (4) singularidades dos sujeitos: quais são as demandas e as necessidades?; (5) autorização para o sentir: reflexões sobre ser terapeuta ocupacional e o manejo dos sentimentos na relação terapêutica; (6) ações em terapia ocupacional: responsabilidades, conceitos e instrumento de trabalho.

\section{1) Reflexões iniciais: o campo - do acolhimento às questões institucionais}

Quando o campo de prática recebe os estudantes com atividades pedagogicamente organizadas e acolhimento das angústias naturais do processo inicial de formação prática, percebemos que os estudantes sentem-se dispostos para a aprendizagem, reconhecendo seu valor e seu lugar na equipe e na instituição. O contrário pode criar um bloqueio a situações de aprendizagem e esse aspecto precisa ser trabalhado tanto no campo como no GRP.

Como o estudante, nesse ciclo, não é o responsável pelos atendimentos, ele possui um olhar reflexivo aguçado para os problemas que, mesmo com possíveis distorções pela falta de experiência e repertório, podem ser importantes para o campo, para identificar falhas em seu processo de trabalho ou funcionamento, como a precariedade do sistema de saúde, de transporte (que inviabilizam a chegada do usuário ao serviço), problemas institucionais, e críticas sociais amplas relacionadas ao lugar social dos sujeitos-alvo das intervenções. Esse material vai sendo retomado nos encontros posteriores de modo a tomar forma e possibilitar construções de conhecimento.

2) As pessoas sob cuidado em terapia ocupacional: expectativas para a vida, familiares e sociais, aspectos emocionais, enfrentamento de situações adversas, impacto no cotidiano

O primeiro tema trabalhado é "Sujeito e PopulaçãoAlvo em Terapia Ocupacional". Nos grupos da Infância e Juventude é possível observar o foco dado pelos estudantes aos déficits, dificuldades, patologias, demandas sociais, educacionais, familiares e contextuais.

Porém, inicialmente, busca-se problematizar as temáticas "infância" e "adolescência" para além das possíveis dificuldades vivenciadas pelos sujeitos-alvo por meio de exercícios que visam a aproximação dos estudantes ao universo infantojuvenil, tanto contemporâneo como a partir de uma revisão da própria infância e adolescência. Busca-se olhar para a população-alvo por meio de seus interesses, fazeres, atividades cotidianas, desejos, potencialidades e contextos de vida.

Algumas hipóteses construídas abarcam: (a) as crianças têm seu processo de desenvolvimento influenciado pelos contextos de vida, em especial, na família, escola e comunidade, (b) estão em processo contínuo de crescimento, apresentando potencialidades de ordem motora, cognitiva e social, (c) as dificuldades apresentadas são colocadas a partir da demanda, por um padrão aceito socialmente, e (d) as demandas apresentadas são colocadas, na maioria das vezes, pelos adultos que vivem ao seu redor, porém, elas também podem/devem ser ouvidas e vistas em suas particularidades.

Algumas das questões que já emergiram nos grupos foram: "como a família e a escola podem influenciar o 
processo de crescimento de crianças e adolescentes?"; "quais são as potencialidades apresentadas pelas crianças observadas pelo terapeuta ocupacional?"; "como as crianças costumam se comunicar e se expressar?"; "qual é o papel da escola no processo de desenvolvimento das crianças e adolescentes?".

Nos grupos de Adulto e Idoso, o trabalho sobre as narrativas desse tema levanta hipóteses sobre as diversas problemáticas que os sujeitos-alvo enfrentam, principalmente sob duas perspectivas (a) a das construções sociais em torno das diferentes problemáticas (deficiências físicas, doença mental, marginalidade e vulnerabilidade social) e (b) da perspectiva subjetiva-individual (de sujeitos e de familiares) para o enfrentamento da situação problemática.

Algumas questões de aprendizagem decorrentes dessas hipóteses abarcam perguntas a serem exploradas com os próprios sujeitos das intervenções: "daqui a alguns anos, como você gostaria de estar em relação às suas atividades?"; e busca de material acadêmico-científico que ajude a compreender essas questões: "Qual o impacto social da situação problemática na vida das pessoas e familiares?", "De qual forma a expectativa do sujeito influencia no tratamento em terapia ocupacional?", "O que é capacidade?", "Como as pessoas enfrentam situações novas e perturbadoras?".

\section{3) Onde estamos? O lugar do campo/serviço/instituição}

Em alguns encontros, as questões do campo ou institucionais, temática inicialmente transversal, assumem o centro das reflexões. Para o entendimento do serviço e de sua relação com a rede intersetorial, algumas vezes, utilizamos ferramentas como o fluxograma, que permite visualizar a entrada e saída do sujeito-alvo, processos de encaminhamento, fomentando a construção de uma compreensão do serviço inserido em uma rede de cuidados e das relações político-institucionais.

A crítica institucional pode acontecer na medida em que se compreende o espaço de cuidado, a proposta do serviço e possíveis engessamentos e paralisias institucionais para as quais os estudantes podem tecer críticas, mas também pensar/construir estratégias para retornar ao campo de modo positivo e produtivo. Dos GRP surgem propostas de intervenção institucional, muitas vezes simples e voltadas para problemas específicos, como a construção de um mural para facilitar a comunicação da equipe.

\section{4) Singularidades dos sujeitos: quais são as demandas e as necessidades?}

As reflexões oriundas do segundo tema trabalhado, "processos de identificação de necessidades em terapia ocupacional", levam os estudantes a refletirem para além da situação problemática específica de cada campo, buscando compreender o que os sujeitos desejam. Nesse sentido, eles conseguem elencar processos pelos quais realizam a identificação de necessidades (observação, escuta, diálogo/ entrevista com o sujeito e seus familiares, processo de realização das atividades, protocolos específicos e testes padronizados), mas o que se coloca como central é como o sujeito percebe seus problemas e quais suas expectativas diante do cuidado em terapia ocupacional. No caso da atenção à criança e ao adolescente, as expectativas da família ganham um foco importante nas reflexões, bem como a análise de como a própria criança e/ou adolescente expressa o que espera desse cuidado.

As hipóteses iniciais abarcam: "os contextos de vida são fundamentais no processo de compreensão da vivência do sujeito"; "o trabalho de terapia ocupacional tem um escopo amplo, porque é o ser inteiro e o cotidiano é tudo, o que leva a uma necessidade de escutar para além do problema"; "não é somente a quebra de cotidiano, e o social?"; "acho que os pacientes querem voltar a fazer como antes. Mas precisamos olhar para o que é possível, para potencializar fazeres"; "com o tempo as necessidades se modificam". Dessas hipóteses, algumas questões de aprendizagem elaboradas foram: "qual a diferença entre necessidade e demanda?"; "o que a literatura indica sobre a avaliação em Terapia Ocupacional"; "como identificar necessidades quando o tempo é curto?"; "como se identificam necessidades diferentes das que o sujeito traz?"; "Qual a definição de cotidiano?".

\section{5) Autorização para o sentir: reflexões sobre ser terapeuta ocupacional e o manejo dos sentimentos na relação terapêutica}

Na discussão sobre a identificação de necessidades, quando se caminha em direção da construção de sentidos dos sujeitos, os estudantes relatam situações vividas na prática e abrem espaço para serem questionados sobre os sentimentos dos sujeitos diante do que lhe acontece/ aconteceu e como eles percebem e lidam com seus sentimentos no contato com o outro. Nessa direção, o vínculo aparece como uma palavra que parece ser a solução para todas as respostas.

A hipótese inicial "nada é possível sem vínculo", abre espaço para questões de aprendizagem que possibilitam aprofundar as reflexões sobre a relação terapêutica na terapia ocupacional: "o que é vínculo?"; "como os autores em terapia ocupacional tem abordado a relação terapêutica?". Nesse processo, também fomentamos a discussão sobre o que os estudantes 
sentem no contato com o outro, percebendo e nomeando sentimentos, refletindo sobre o que gera tais sentimentos, sejam eles ligados à (a) situação de aprendizagem inicial, como insegurança, medo, crítica excessiva, (b) a sentimentos por situações similares às dos sujeitos, (c) sentimentos provenientes da relação com o outro, (d) sentimentos e reflexões relacionados ao que é ser um(a) bom(a) terapeuta ocupacional.

As hipóteses e questionamentos giram em torno de que "cada pessoa tem um modo de se relacionar na vida"; "estar em terapia ocupacional, precisar de ajuda, tudo isso gera sentimentos e emoções diversas", gerando questões de aprendizagem como: "como os sentimentos do terapeuta ocupacional podem ser usados como fonte de informação sobre as necessidades dos sujeitos?"; "como se constrói a relação terapêutica em terapia ocupacional?"; "como identificar o processo de transferência e contratransferência?”.

\section{6) Ações em terapia ocupacional: responsabilidades, conceitos e instrumento de trabalho}

Na prática com adultos, essa temática vem surgindo atrelada à construção da responsabilidade da(o) terapeuta ocupacional na condução do processo terapêutico, em uma construção de que o processo é colaborativo, mas as ações precisam ser responsáveis. Esse tema é trabalhado por meio da discussão das narrativas e experiências do campo e de questões de aprendizagem que normalmente partem para explorações práticas e entrevistas com profissionais. É comum aparecerem ações para o cuidado do corpo e das emoções, mas nem sempre focadas na inserção e participação social, gerando questões de aprendizagem como: "como o social aparece nas intervenções em meu campo de prática?", "o que é o social para a terapia ocupacional?".

No grupo de estudantes da Infância e Adolescência, o interesse pelas atividades emerge fortemente nas narrativas e reflexões. Nessa direção, temáticas como o "brincar" e o papel das atividades na ação da(o) terapeuta ocupacional são abordadas a partir de diferentes disparadores. Reforça-se que a discussão transcende a especificidade dos campos de atuação, mas focaliza a terapia ocupacional e seu instrumento de ação. Assim, algumas questões de aprendizagem nessa temática abarcam: "qual é o papel da atividade na ação do terapeuta ocupacional junto a crianças e adolescentes?", "como o brincar pode ser explorado nos diferentes campos de prática?", "como compreender o conceito de atividade no campo da assistência da terapia ocupacional junto a crianças e adolescentes?”.

\section{DISCUSSÃO}

Partindo da integração entre a vivência de experiências nos campos de atuação e o espaço para reflexão, os GRP distanciam-se das demandas urgentes da prática ${ }^{10}$ - trabalhadas especificamente nas supervisões do campo ${ }^{11}$. Além disso, apresentam-se como proposta pedagógica que demanda construção conjunta de estratégias formativas entre os diferentes atores (professores facilitadores da reflexão, professores dos campos de prática, professores das outras unidades educacionais/disciplinas do curso no referido semestre/ano), de modo a potencializar a aprendizagem da prática profissional.

A Espiral Construtivista ${ }^{8}$ possibilita flexibilidade para que as experiências práticas sejam potentes para o processo reflexivo que, por sua vez, instrumentaliza novas experimentações no campo prático a partir das novas compreensões construídas ${ }^{1}$. Assim, conceitos trabalhados anteriormente em outras disciplinas (sujeito em sua totalidade, cotidiano, vínculo) podem ser aprofundados e trabalhados teoricamente na medida em que se compreende sua importância na prática profissional (aprendizagem significativa).

Desse modo, a problemática da nossa população alvo é trabalhada na inter-relação entre a subjetividade dos sujeitos, nas vivências de situações particulares que implicam mudanças em seu cotidiano ou limitações de acordo com a posição social que ocupam, e as construções sociais em torno dessa situação problemática, demarcando posições de exclusão. Abrimos espaços para reflexões sobre as demandas da sociedade, das instituições, dos projetos, mas, principalmente, a demanda e o desejo dos sujeitos, que podem ser diferentes das necessidades identificadas a partir do saber técnico narrativo-científico ${ }^{12,13}$. Essas reflexões fomentam questionamentos sobre ações em terapia ocupacional que vislumbrem a construção de uma história compartilhada, na qual ambos possam investir ${ }^{14}$.

Nesse processo, ao explorar racionalidades e emoções $^{8}$, os estudantes experimentam pensamentos e sentimentos reais como terapeutas ocupacionais e poder falar sobre essas vivências é avaliado como algo realmente importante. Ter um momento garantido na estrutura curricular para a reflexão do seu próprio "estudo" parece gerar maior satisfação entre os estudantes, na medida em que eles se percebem como pessoas com sentimentos, conceitos e valores que devem ser revisitados e, algumas vezes, transformados para o exercício da prática profissional ${ }^{12}$.

No GRP, o foco é a terapia ocupacional e os campos de atuação agem como cenários da vida prática-profissional. 
Assim, cria-se espaço para refletir sobre as intervenções e sobre seus diferentes objetivos, sobre o lugar dado à inserção e participação social dos sujeitos, individuais e coletivos, que estão sob nosso cuidado, e a discussão da atividade/ brincar enquanto algo que, de forma contextualizada, se integra ao sujeito delineando seu cotidiano.

\section{CONSIDERAÇÕES FINAIS}

O presente trabalho buscou retratar a experiência que vem sendo desenvolvida junto aos estudantes do terceiro ciclo do eixo educacional de Prática Simulada e Supervisionada do curso de graduação em Terapia Ocupacional da UFSCar. A partir dessa experiência é possível observar que o processo ensino-aprendizagem da prática envolve um constante e intenso desafio na relação pensamento-prática, ação-reflexão-ação, essência da atividade profissional em terapia ocupacional. Nesse sentido, o GRP tem se configurado como um espaço no qual os estudantes têm a oportunidade de concretizar um processo de aprendizagem significativa. Avaliamos que essa vivência tem possibilitado crescimento e amadurecimento profissional.

O presente trabalho advém do relato da experiência e reflexões de docentes, portanto, vale apontar a demanda por estudos sistematizados que se debrucem sobre esta temática para melhor subsidiar discussões relativas à formação profissional no campo da saúde, educação e assistência social que se constituem de híbridas e complexas necessidades. Defendemos que o processo de ação-reflexão-ação não seja apreciado apenas como uma estratégia educacional a mais, dado que agencia aprendizagens essenciais para a prática profissional. Ao possibilitar a reflexão sobre a prática, tal processo contribui para a construção de uma identidade profissional sólida, por reconhecer características comuns de sua ação, e flexível, por se permitir construir com sujeitos singulares em contextos complexos.

Agradecimentos: Agradecimentos à Profa. Dra. Lilian Vieira Magalhães, professora do Departamento de Terapia Ocupacional da UFSCar e professora emérita da University of Western Ontario, pela constante colaboração com a busca e disponibilização de artigos científicos.

\section{REFERÊNCIAS}

1. Schön D. Educando o profissional reflexivo: um novo design para o ensino e a aprendizagem. Porto Alegre: Artes Médicas; 2000.

2. Brasil. Ministério da Educação. Diretrizes Curriculares Nacionais do curso de graduação em Terapia Ocupacional. Brasília; 2002 [citado 30 jul. 2013]. Disponível em: http://portal.mec.gov.br/cne/arquivos/ pdf/ CES062002.pdf.

3. Universidade Federal de São Carlos (UFSCar). Projeto Pedagógico do Curso de Graduação em Terapia Ocupacional. São Carlos; 2015 [citado 10 ago. 2016]. Disponível em: http://www2.ufscar.br/interface_frames/ index.php?link=http://www.prograd.ufscar.br/cursos.php.

4. Universidade Federal de São Carlos (UFSCar). Projeto Pedagógico do Curso de Graduação em Terapia Ocupacional. São Carlos; 2007.

5. Universidade Federal de São Carlos (UFSCar). Projeto Pedagógico do Curso de Graduação em Terapia Ocupacional. São Carlos; 2011.

6. Della Barba PCS, Silva RF, Joaquim RHVT, Brito CMD. Formação inovadora na graduação em Terapia Ocupacional.
Interface (Botucatu). 2012;16(42):829-42. doi: 10.1590/ S1414-32832012000300019.

7. World Federation of Occupational Therapists. Revised minimum standards for the education of occupational therapists. Bethesda; 2002.

8. Lima VV. Espiral construtivista: uma metodologia ativa de ensino-aprendizagem. Interface (Botucatu). 2016;21(61):421-34. http://dx.doi.org/10.1590/180757622016.0316 .

9. Cardoso PT, Cruz DMC, Della Barba PCS. Metodologias Ativas de Aprendizagem: potencial dos grupos reflexivos da prática na formação de terapeutas ocupacionais. REFACS Rev Familia Ciclos Vida Saude Contexto Social. 2015;3(Supl 1):151-8. doi: 10.18554/refacs. v3i3.1096.

10. Marcolino TQ, Lourenço GF, Reali AMMR. "Isso eu levo para a vida!": aprendizagem da prática profissional em uma comunidade de prática. Interface (Botucatu). 2017;21(61):411-20. doi: 10.1590/1807-57622016.0099.

11. Signe PB, Lourenço GF, Marcolino TQ. Ensino e aprendizagem da prática profissional: o caso dos supervisores 
de estágio do curso de graduação em Terapia Ocupacional da UFSCar. Rev Ter Ocup Univ São Paulo. 2017; 28(1):2735. doi: 10.11606/issn.2238-6149.v28i1p27-35.

12. Bonsaksen T. Predictors of academic performance and education programme satisfaction in occupational therapy students. Br J Occup Ther. 2016;79(6):361-7. doi: $10.1177 / 0308022615627174$.

Recebido em: 27.09.2016

Aceito em: 27.07.2017
13. Marcolino TQ. Reflexões sobre a investigação do raciocínio clínico em terapia ocupacional: o caso do Método Terapia Ocupacional Dinâmica. Cad Ter Ocup UFSCar. 2014;22(3):635-42. doi: 10.4322/cto.2014.086.

14. Mattingly C, Fleming MH. Clinical reasoning: forms of inquiry in a therapeutic process. Philadelphia: F. A. Davis Company; 1994. 